\title{
EVEN COMPOSITIONS OF ENTIRE FUNCTIONS AND RELATED MATTERS
}

\author{
ALAN HORWITZ \\ (Received 1 August 1996; revised 24 January 1996) \\ (Dedicated to the memory of Lee Rubel.)
}

Communicated by P. C. Fenton

\begin{abstract}
We examine when the composition of two entire functions $f$ and $g$ is even, and extend some of our results to cyclic compositions in general. If $p$ is a polynomial, then we prove that $f \circ p$ is even for a non-constant entire function $f$ if and only if $p$ is even, odd plus a constant, or a quadratic polynomial composed with an odd polynomial. Similar results are proven for odd compositions. We also show that $p \circ f$ can be even when $f$ and no derivative of $f$ are even or odd, where $p$ is a polynomial. We extend some results of an earlier paper to cyclic compositions of polynomials. We also show that our results do not extend in general to rational functions or polynomials in two variables.
\end{abstract}

1992 Mathematics subject classification (Amer. Math. Soc.): 30B 10.

Keywords and phrases: Entire, even, composition.

\section{Introduction}

Let $f(z)$ and $g(z)$ be entire functions with their composition $f \circ g$ even. What can be said about $f$ and $g$ ? Also, given certain conditions on $f$ and/or $g$, can $f \circ g$ be even? There are numerous examples which show that $f \circ g$ can be even, while neither $f$ nor $g$ is even. In [3] we proved that if $f$ and $g$ are non-constant polynomials with $g(0)=0$ and $g$ neither even nor odd, then $f \circ g$ cannot be even. It was also shown that if $f$ and $g$ are polynomials, $f$ not constant, $g(0)=0$, and $f \circ g$ is even, then $f$ or $g$ must be even. These results do not hold for entire functions in general, or for rational functions in general.

Call an entire function $g(z)$ right pseudo-even (RPE) if there is a non-constant entire function $f(z)$ such that $f \circ g$ is even. Of course, every even or odd entire function

(C) 1997 Australian Mathematical Society 0263-6115/97\$A2.00+0.00 
is RPE, but there are entire functions $g$ which are RPE, while $g$ (or any derivative of $g$ ) is neither even nor odd. An example is $g(z)=\sin (z)+\cos (z)$. In Section 1 we completely characterize the RPE polynomials $p(z)$. Using a result from [1], $p$ must be either even, an odd polynomial plus a constant, or a quadratic polynomial composed with an odd polynomial $s(z)$ (Theorem 2.2). For the latter case, a simple example is $p(z)=\left(z^{2}+2 z\right) \circ s(z), f(z)=\cos (2 \pi \sqrt{z+1})$. Similar results are given for when $f \circ p$ is odd (Theorem 2.3).

Call an entire function $f(z)$ left pseudo-even (LPE) if $f \circ g$ is even for some entire function $g(z)$ which is not even. Every even entire function is LPE, but there are also LPE entire functions which are not even. An example is the polynomial $f(z)=\left(z^{2}+2 z\right)^{2}$. In Section 2 we discuss the more general question of when $p \circ g$ can be even, where $g(z)$ is entire and $p(z)$ is a polynomial. However, we are not able to give a complete characterization of the LPE polynomials, as we do for the RPE polynomials. We can prove (Theorem 3.2) that if $g$ is a transcendental entire function with finitely many zeroes, which is neither even nor odd, then $p \circ g$ cannot be even for any non-constant polynomial $p$. We can prove the simple result that $p(z)=z^{n}, n$ odd, cannot be LPE(see Lemma 3.4). It is interesting to note, however, that it is possible for an odd entire function to be LPE. For example, $f(z)=\sin \left(\frac{1}{2} \pi z\right)$ is LPE since $f \circ g$ is even, where $g(z)=1-\sin \left(\frac{1}{2} \pi z\right)$.

In Section 3 we extend some of our results to cyclic compositions. Let $N$ be a prime number, and let $\omega$ be a primitive $N$ th root of unity. If $f(z)$ is analytic at 0 , and $f(z)=z^{k} \sum_{j=0}^{\infty} a_{j} z^{j N}$, then $f$ is called cyclic $(\bmod N)$. Then $f$ is cyclic $(\bmod 2)$ if and only if $f$ is even or odd. If $N \geq 3, f$ is entire, and $p$ is a polynomial, then necessary and sufficient conditions for $f \circ p$ to be cyclic $(\bmod N)$ are more restrictive than for the even or odd case (Theorem 4.5).

In Section 4 we discuss even and odd compositions of rational functions, where the results for polynomials do not extend in general. For example there are rational functions $f(z)$ and $g(z)$ such that $f(0)=g(0)=0, f \circ g$ is even, but neither $f$ nor $g$ are even. It is indeed possible for $f \circ f$ to be even when $f$ is not even $(f(0) \neq 0)$. We do not know if this is possible for the cyclic case in general.

In Section 5 we discuss even compositions of polynomials in two variables. Again, the results do not extend in general. There are polynomials $P(z, w), Q(z)$, and $R(z), P(0,0)=0, Q(0)=R(0)=0$, and $P, Q$, and $R$ neither even nor odd, with $P(Q(z), R(z))$ even. One can prove, however, that if $Q(0,0)=0$ and $P(Q(z, w))$ is even, where $P$ is a polynomial in one variable, then $P$ or $Q$ must be even. Related questions along these lines involve homogeneous or symmetric compositions of polynomials in two or more variables. 


\section{Entire functions composed with a polynomial}

Let $f$ be an entire function and $p$ a polynomial. We shall prove that if $f \circ p$ is even, then either $p$ is even, odd plus a constant, or a quadratic polynomial composed with an odd polynomial. First we need the following lemma.

LEMMA 2.1. Suppose that $g$ is analytic at 0 , and $g$ is neither even, nor odd plus a constant (that is, $g^{\prime}$ is neither even nor odd). Then there are no constants $a$ and $b$ such that $g(z)=a g(-z)+b$ for all $z$.

PROOF. Write $g(z)=\sum_{k=0}^{\infty} a_{k} z^{k}$, and let $m$ and $n$ be distinct positive integers with $m$ odd, $n$ even, and $a_{m} \neq 0 \neq a_{n}$. Then the expansion of $g(z)-a g(-z)$ about 0 contains the terms $a_{m}(1+a) z^{m}$ and $a_{n}(1-a) z^{n}$, and hence no value of $a$ can make $g(z)-a g(-z)$ a constant.

THEOREM 2.2. Let $p$ be a polynomial. Then there exists a non-constant entire function $f$ such that $f \circ p$ is even if and only if one of the following holds:

(A) $p$ is even.

(B) $p(z)=s(z)+k$, where $s$ is odd and $k$ is a constant.

(C) $p(z)=(s(z)+d)^{2}+k$, where $s$ is odd and $d$ and $k$ are constants, $d \neq 0$.

PROOF. We can assume $p \neq 0$, since $p=0$ is equivalent to (A), (B), and (C) all holding. First we prove that if $f \circ p$ is even, then one of (A), (B), or (C) must hold. Assume first that neither (A) nor (B) holds, and let $q(z)=p(-z)$. Then

$$
f(p(z))=f(q(z)), \quad \forall z .
$$

By [1],

$$
p(z)=a q(z)+b
$$

or

$$
p(z)=r^{2}(z)+k, \quad q(z)=(r(z)+c)^{2}+k .
$$

By Lemma 2.1, (2.3) must hold. Now $p(-z)=r^{2}(-z)+k=(r(z)+c)^{2}+k$ implies $r^{2}(-z)=(r(z)+c)^{2}$, which implies $r(-z)= \pm(r(z)+c)$. Thus $-r^{\prime}(-z)= \pm r^{\prime}(z)$, implying $r^{\prime}$ is either even or odd. If $r^{\prime}$ is odd, then $r$ is even, which implies that $p$ is even. That contradicts the assumption that (A) does not hold. Hence $r^{\prime}$ is even which implies $r(z)=s(z)+d$, where $s$ is an odd polynomial. Hence $p(z)=(s(z)+d)^{2}+k$, 
and (C) holds. Now if (A) and (C) do not hold, the above argument shows that $p$ must satisfy (2.2). By Lemma 2.1, $p(z)=s(z)+k$, where $s$ is odd, and hence (B) holds. Finally, if (B) and (C) do not hold, then Lemma 2.1 and the argument above show that $p$ must be even, and hence (A) holds.

To prove sufficiency, clearly if (A) or (B) hold, then one can always find a nonconstant entire function $f$ such that $f \circ p$ is even. If (C) holds, one can choose $f(z)=\cos \left(2 \pi d^{-1} \sqrt{z-k}\right)$. Then $f\left((z+d)^{2}+k\right)=\cos (2 \pi z / d)$, which is even. Hence $f\left((s(z)+d)^{2}+k\right)$ is also even.

REMARK 2.1. (A) and (B) are equivalent to $p(z)-p(0)$ being even or odd.

REMARK 2.2. Theorem 2.2 gives a complete characterization of the RPE polynomials.

An odd version of Theorem 2.2 also follows. The proof is similar to the proof of Theorem 2.2 and we omit it.

THEOREM 2.3. Let $p$ be a polynomial. Then there exists a non-constant entire function $f$ such that $f \circ p$ is odd if and only if one of the following holds:

(A) $p$ is odd.

(B) $p(z)=(s(z)+d)^{2}+k$, where $s$ is odd and $d$ and $k$ are constants, $d \neq 0$.

REMARK 2.3. If (B) of Theorem 2.3 holds, choose $f(z)=\cos \left(\frac{1}{2} \pi \sqrt{z-k} / d\right)$ to make $f \circ p$ odd.

EXAMPLE 2.1. Let $p(z)=z^{k}+z$, where $k \geq 4, k$ even. Then it is not hard to show that (C) of Theorem 2.2 (or (B) of Theorem 2.3) does not hold (the other conditions are trivial). Hence there is no non-constant entire function $f$ such that $f \circ p$ is even or is odd. There is, however, a function $f$ analytic at 0 such that $f \circ p$ is even (or odd). Since $p$ has an inverse in a neighborhood of $z=0$, just let $f=x^{2} \circ p^{-1}$ (or $\left.p^{-1}\right)$.

\section{Polynomials composed with an entire function}

Let $p$ be a non-constant polynomial and $f$ an entire function. As we show below, $p \circ f$ can be even, even when $f$ is neither even, nor odd plus a constant. It would be nice to have a theorem similar to Theorems 2.2 and 2.3 which characterizes when $p \circ f$ is even, but we are not able to do this. One can easily show that if $p \circ f$ is even, then there must be a polynomial relation between the even and odd parts of $f$, but this is not sufficient. For example, let $f(z)=e^{z}=\sinh (z)+\cosh (z)$. There is 
a polynomial relation between $\sinh (z)$ and $\cosh (z)$, but no non-constant polynomial in $e^{z}$ can be even. We prove a more general result than this below. However, certain polynomial relations in the even and odd parts of $f$ do imply that $p \circ f$ can be even.

THEOREM 3.1. Let $f(z)=E(z)+O(z)$, where $E$ is even, $O$ is odd, and $E^{2}+O^{2}=$ 1 for all $z$. Then $p \circ f$ is even, where $p(z)=z^{4}-2 z^{2}$.

ProOF. $(E+O)^{2}-1=2 O E$, which is odd. Hence $\left((E+O)^{2}-1\right)^{2}$ being even implies $\left((E+O)^{2}-1\right)^{2}-1=(E+O)^{4}-2(E+O)^{2}$ is even as well.

EXAMPLE 3.1. Let $f(z)=\cos (z)+\sin (z)$. Then

$$
p(\sin (z)+\cos (z))=-4 \cos ^{4} z+4 \cos ^{2} z-1 .
$$

The example above can be modified so that $f(0)=0$ and $p$ (as well as $f$ ) is not even. Just let $f(z)=\cos (z)+\sin (z)-1$, and let $q(z)=z^{2}+2 z$. Then $q(f(z))=2 \cos z \sin z$, which is odd. Hence $p(f(z))$ is even, where $p=q^{2}$. Note also that $f$ is neither even, nor odd plus a constant. However, $f$ does have infinitely many zeros. This must be the case, as the following theorem shows.

THEOREM 3.2. Suppose that $f$ is a transcendental entire function with finitely many zeroes in the plane, and let $p$ be a non-constant polynomial.

(A) If $f$ is neither even nor odd, then $p \circ f$ cannot be even.

(B) If $f$ is not odd, then $p \circ f$ cannot be odd.

Before proving Theorem 3.2, we need the following theorem first stated by Borel. The first complete proof was given by Nevanlinna [4].

THEOREM 3.3 (Borel [2]). Let $a_{i}(z)$ be an entire function of order $\rho$, let $g_{i}(z)$ also be entire and let $g_{j}(z)-g_{i}(z)(i \neq j)$ be a transcendental function or polynomial of degree higher than $\rho$. Then $\sum_{i=1}^{n} a_{i}(z) e^{g_{i}(z)}=a_{0}(z)$ holds only when $a_{0}(z)=a_{1}(z)=$ $\cdots=a_{n}(z)=0$.

PROOF OF THEOREM 3.2. We prove (A), the proof of (B) following in a similar fashion. Since $f$ is transcendental and has finitely many zeroes, $f(z)=Q(z) e^{g(z)}$, where $Q$ is a polynomial and $g$ is a non-constant entire function. Write $p(z)=$ $\sum_{i=0}^{n} a_{i} z^{i}, a_{n} \neq 0, n \geq 1$. Then $p(f(z))=\sum_{i=0}^{n} a_{i}(Q(z))^{i} e^{i g(z)}$. Hence

$$
p(f(z))-p(f(-z))=\sum_{i=0}^{n} a_{i}(Q(z))^{i} e^{i g(z)}-\sum_{i=0}^{n} a_{i}(Q(-z))^{i} e^{i g(-z)} .
$$

Now suppose that $p \circ f$ is even. 
Case 1: $g$ is even. Then, since $p \circ f$ is even,

$$
\sum_{i=0}^{n} a_{i} e^{i g(z)}\left((Q(z))^{i}-(Q(-z))^{i}\right)=0
$$

By Theorem 3.3 with $a_{i}(z)=a_{i}(Q(z))^{i}-(Q(-z))^{i}, i \geq 1$ and $a_{0}=0$, which are entire functions of order $0, a_{i}(Q(z))^{i}-(Q(-z))^{i}=0$ for all $i$. Since $a_{n} \neq$ $0,(Q(z))^{n}-(Q(-z))^{n}=0$, which implies that $Q$ is either even or odd (see Lemma 3.4 below). This contradicts the fact that $f$ is neither even nor odd.

Case 2: $g(z)=h(z)+C$, where $h$ is odd and non-zero, and $C$ is a constant. Then, since $p \circ f$ is even,

$$
\sum_{i=0}^{n} a_{i} e^{i C}(Q(z))^{i} e^{i h(z)}-\sum_{i=0}^{n} a_{i} e^{i C}(Q(-z))^{i} e^{-i h(z)}=0 .
$$

Again, by Theorem 3.3, with $a_{i}(z)=a_{i} e^{i C}(Q(z))^{i}$ or $a_{i} e^{i C}(Q(-z))^{i}, i \geq 1$ and $a_{0}=0, a_{i} e^{i C}(Q(z))^{i}=0$ for all $i$. Hence $(Q(z))^{n}=0$, which implies that $Q=0$, which contradicts the fact that $f$ cannot be 0 .

Case 3: $g$ is neither even, nor odd plus a constant.

By Lemma 2.1, $k g(z)-j g(-z)$ cannot be a constant for $k$ and $j$ positive integers. Since $p \circ f$ even implies that

$$
\sum_{i=0}^{n} a_{i}(Q(z))^{i} e^{i g(z)}-\sum_{i=0}^{n} a_{i}(Q(-z))^{i} e^{i g(-z)}=0,
$$

by Theorem 3.3, with $a_{i}(z)=a_{i}(Q(z))^{i}$ or $a_{i}(Q(-z))^{i}, i \geq 1$ and $a_{0}=0, a_{i}(Q(z))^{i}=$ 0 for all $i$. Hence $(Q(z))^{n}=0$, which implies that $Q=0$, which contradicts the fact that $f$ cannot be 0 .

REMARK 3.1. Theorem 3.2 does not follow in general if $f$ is not transcendental, as the simple example $f(z)=z-1$ and $p(z)=(z+1)^{2}$ or $p(z)=(z+1)^{3}$ shows. However, (A) does follow for polynomials with the stronger assumption that $f$ is neither even nor odd plus a constant. (B) follows for polynomials if $f$ is not odd plus a constant. This follows from [3] or from Theorem 4.3 (B) in the next section.

Finally we end this section by proving that if $p(z)=z^{n}$ and $f(z)$ is neither even nor odd, then $p \circ f$ cannot be even.

LEMMA 3.4. Let $f$ be an entire function, and suppose that $f^{n}(z)=(f(z))^{n}$ is even for some positive integer $n$. Then $f$ must be even or odd. 
PROOF. If $f(z))^{n}=(f(-z))^{n}$ then $(f(z) / f(-z))^{n}=1$. Thus $f(z) / f(-z)=$ $e^{2 \pi k i / n}$, where $k \in Z_{+}$depends on $z$, and $f(-z) \neq 0$. By continuity, $f(z) / f(-z)=$ $e^{2 \pi k i / n}, k$ independent of $z$. Hence $f(z)=e^{2 \pi k i / n} f(-z)$. Let $f(z)=E(z)+O(z)$, where $E$ is even and $O$ is odd. Then $\left(1-e^{2 \pi k i / n}\right) E(z)=\left(-1-e^{2 \pi k i / n}\right) O(z)$. If $e^{2 \pi k i / n} \neq 1$ or -1 , then $E(z)=O(z)=0$ and $f=0$. If $e^{2 \pi k i / n}=1$, then $O(z)=0$ and $f$ is even. If $e^{2 \pi k i / n}=-1$ ( $n$ is even), then $E(z)=0$ and $f$ is odd.

REMARK 3.2. Lemma 3.4 implies that $z^{n}, n$ odd, cannot be LPE.

\section{Cyclic compositions}

Let $N$ be a prime number, and let $\omega$ be a primitive $N$ th root of unity. Let $C=$ $\bigcup_{k=0}^{N-1} C_{k}$, where

$$
C_{k}=\left\{f \text { analytic at } 0: f(\omega z)=\omega^{k} f(z)\right\}, \quad k=0, \ldots, N-1 .
$$

We call the functions in $C$ cyclic $(\bmod N)$. Note that

$$
f \in C_{k} \text { implies } f(z)=z^{k} \sum_{j=0}^{\infty} a_{j} z^{j N} .
$$

Of course, $C$ and $C_{k}$ depend on $N$, but we suppress this in our notation. For $N=2$, elements of $C_{0}$ are even functions and elements in $C_{1}$ are odd functions, analytic at 0 . In [3] we proved that if $p$ and $q$ are polynomials, $q(0)=0, q$ neither even nor odd, then $p \circ q$ is neither even (if $p$ not constant) nor odd. As a corollary, if $q(0)=0$ and $p \circ q$ is even, then $p$ and/or $q$ must be even.

A similar result follows for the odd case. We extend this result now to cyclic functions.

PROPOSITION 4.1. Let $p$ and $q$ be polynomials with $p$ not constant, $q(0)=0$, and $q \notin C$. Then $p \circ q \notin C$.

Before proving Proposition 4.1 we need the following lemma.

LEMMA 4.2. Let $m, n$, and $r$ be positive integers with $n \equiv 0(\bmod N), r \not \equiv m$ $(\bmod N)$. Then $m(n-1)+r \not \equiv 0(\bmod N)$.

PROOF. $n=k N, r=l N+i, m=s N+j$, with $i \neq j$. Then $m(n-1)+r=(s N+j)(k N-1)+l N+i \equiv(i-j) \quad(\bmod N) \not \equiv 0 \quad(\bmod p)$. 
PrOOF OF PROPOSITION 4.1. Let $m=\operatorname{deg} q, n=\operatorname{deg} p$, and assume without loss of generality that $p$ is monic. Now write

$$
p(z)=\left(z-\alpha_{1}\right) \cdots\left(z-\alpha_{n}\right), \quad q(z)=\sum_{k=1}^{m} a_{k} z^{k} .
$$

Then

$$
p(q(z))=\left(\sum_{k=1}^{m} a_{k} z^{k}-\alpha_{1}\right) \cdots\left(\sum_{k=1}^{m} a_{k} z^{k}-\alpha_{n}\right) .
$$

We prove first that $p \circ q \notin C_{0}$.

Case $1 m \equiv 0(\bmod N)$. Let $r$ be the highest power of $z$ in $q, r \not \equiv 0(\bmod N)$. Then it follows easily from (4.1) that $m(n-1)+r$ is the highest power of $z$ in $p \circ q \not \equiv 0$ $(\bmod N)$, and the coefficient of $z^{m(n-1)+r}$ is $n a_{m}^{n-1} a_{r} \neq 0$. Hence $p \circ q \notin C_{0}$.

Case $2 m \not \equiv 0(\bmod N)$. Suppose that $p \circ q \in C_{0}$. We shall derive a contradiction. If $\operatorname{deg}(p q)=m n \equiv 0(\bmod N)$ then $n \equiv 0(\bmod N)$. Let $r$ be the highest power of $z$ in $q, r \not \equiv m(\bmod N)$. Note that $q(0)=0$ implies $r>0$, and thus there is no cancellation of $z^{r}$ with any $\alpha_{j}$ in (4.1). By Lemma 4.2, $m(n-1)+r \not \equiv 0(\bmod N)$. Now $s>r$ implies $s \equiv m(\bmod N)$. Also, by (4.1), any power of $z$ in $p \circ q$ larger than $m(n-1)+r$ has the form $s_{1}+\cdots+s_{n}$, each $s_{j}>r$. Now suppose that $m \equiv k$ $(\bmod N)$. Then

$$
s_{1}+\cdots+s_{n} \equiv n k \quad(\bmod N) \equiv 0 \quad(\bmod N) .
$$

Hence $m(n-1)+r$ is the largest power of $z$ in $p \circ q$ not congruent to $0(\bmod N)$. Since the coefficient of $z^{m(n-1)+r}$ is $n a_{m}^{n-1} a_{r} \neq 0, p \circ q \notin C_{0}$. This is a contradiction, and hence $p \circ q \notin C_{0}$.

Now suppose that $p \circ q \in C_{j}$ for some $j$. Then $p^{N} \circ q=(p \circ q)^{N} \in C_{0}$, which contradicts the case just proven.

REMARK 4.1. Proposition 4.1 does not follow if $q(0) \neq 0$. For example, let $p(z)=(z+1)^{N}, q(z)=z-1$. Also, it is trivial that Proposition 4.1 does not follow if $N$ is not prime.

REMARK 4.2. The proof of Proposition 4.1 shows that $q(0)=0$ is only needed for case 2, where $\operatorname{deg}(q) \not \equiv 0(\bmod N)$.

THEOREM 4.3. Let $p$ and $q$ be polynomials with $p \circ q \in C$.

(A) Suppose $q(0)=0$. Then $q \in C$ or $p \in C$. Also, if $p$ is not constant and $q \notin C_{0}$, then $p \in C$ and $q \in C$. Finally, if $p \circ q \in C_{0}$, then $p \in C_{0}$ or $q \in C_{0}$.

(B) Suppose $q(0) \neq 0$. If $p$ is not constant and $q \notin C_{0}$, then $q(z)=r(z)+q(0)$, $p(z)=s(z-q(0))$ where $r \in C, s \in C$. 
ProOF. To prove (A), if $p$ is constant, then $p \in C$. If $p$ is not constant and $q \notin C$, then $p \circ q \notin C$ by Proposition 4.1. Hence $q \in C$. Now suppose that $p$ is not constant. We just showed that $q \in C$. Now suppose that $q \in C_{j}, j \geq 1$ and $p \circ q \in C_{k}$. Then

$$
p(q(\omega z))=\omega^{k} p(q(z)) \quad \text { and } \quad p(q(\omega z))=p\left(\omega^{j} q(z)\right)
$$

and hence $p\left(\omega^{j} u\right)=\omega^{k} p(u)$ for any complex number $u$. Choose $r$ so that $r j \equiv 1$ $(\bmod N)$. Then

$$
p(\omega u)=p\left(\omega^{r j} u\right)=\omega^{r k} p(u)
$$

which implies that $p \in C_{i}$, where $r k \equiv i(\bmod N)$. Hence $p \in C$. Finally, suppose that $p \circ q \in C_{0}$. Again, $q \in C$. If $q \notin C_{0}$, then $q \in C_{j}, j \geq 1$. Then

$$
p(q(\omega z))=p(q(z)) \quad \text { and } \quad p(q(\omega z))=p\left(\omega^{j} q(z)\right)
$$

and hence $p(\omega u)=p\left(\omega^{r j} u\right)=p(u), r j \equiv 1(\bmod N)$. Thus $p \in C_{0}$.

To prove (B), let $r(z)=q(z)-q(0)$. If $p$ is not constant, then $p(q(z))=$ $p(r(z)+q(0))=s(r(z))$, where $s(z)=p(z+q(0))$ is a non-constant polynomial. By (A), $r \in C$ and $s \in C$ since $r \notin C_{0}$. Hence $q(z)=r(z)+q(0)$ and $p(z)=s(z-q(0))$ with $r \in C$ and $s \in C$.

REMARK 4.3. It is not true in general that if $p \circ q \in C_{j}$, then $q \in C_{j}$ or $p \in C_{j}$. This only holds in general for $j=0$, as (A) shows. For the case $N=2$, however, it does follow that if $p \circ q$ is odd and $q(0)=0$, then $p$ or $q$ must be odd (see [3]).

THEOREM 4.4. Let $p$ be a polynomial with $p \circ p \in C$.

(A) If $p(0)=0$, then $p \in C$.

(B) If $p \circ p \in C_{0}$, then $p \in C_{0}$.

PROOF. (A) follows immediately from Theorem 4.3 (A) with $p=q$. To prove part $(\mathrm{B})$, note that $\operatorname{deg}(p) \equiv 0(\bmod N)$. By Remark 4.2 following the proof of Proposition 4.1 (with $p=q$ ), $p \in C_{0}$.

REMARK 4.4. (A) does not follow in general if $p(0) \neq 0$. For example, for $N=2$, $p(z)=-z+1$ satisfies $p(p(z))=z$, which is odd, but $p$ is neither odd nor even.

4.1. Cyclic Compositions of Entire Functions with Polynomials For primes $N>$ 2 , we now prove a theorem on cyclic compositions of entire functions with polynomials $p(z)$. The possibilities for $p(z)$ are more restrictive than for Theorems 2.2 and 2.3. 
THEOREM 4.5. Let $p(z)$ be a polynomial and $N \geq 3$ a prime number. Then there exists a non-constant entire function $f(z)$ such that $f \circ p \in C$ if and only if $p(z)-p(0) \in C$.

PROOF. Suppose firstly that $f \circ p \in C_{0}$ for some non-constant entire function $f(z)$, and write $p(z)=\sum_{k=0}^{n} a_{k} z^{k}, a_{n} \neq 0$. Let $\omega$ be a primitive $N$ th root of unity, and let $q(z)=p(\omega z)$. By [1] again, either (2.2) or (2.3) must hold. If (2.2) holds, then $p(\omega z)=\lambda p(z)+\beta$ which implies that

$$
\sum_{k=0}^{n} a_{k} \omega^{k} z^{k}=\lambda \sum_{k=0}^{n} a_{k} z^{k}+\beta .
$$

Hence $\lambda=\omega^{n}$ and $a_{0}=\lambda a_{0}+\beta$ which implies that $\beta=a_{0}\left(1-\omega^{n}\right)$ (Note that if $N \mid n$, then $\lambda=1, \beta=0$, and $p \in C_{0}$ ). Moreover $\lambda a_{j}=\omega^{j} a_{j}, j \geq 1$ implies $\omega^{n}=\omega^{j}$ if $a_{j} \neq 0$, which implies $n \equiv j(\bmod N)$ if $a_{j} \neq 0, j \geq 1$. That implies that $p(z)-p(0) \in C_{k}$ for some $k$.

Now suppose that (2.3) holds. Then

$$
p(z)=r^{2}(z)+k, \quad p(\omega z)=(r(z)+c)^{2}+k
$$

for some constants $c$ and $k$. Hence $r^{2}(\omega z)=(r(z)+c)^{2}$ which implies that $r(\omega z)=$ $\pm(r(z)+c)$ and hence $\omega r^{\prime}(\omega z)= \pm r^{\prime}(z)$. Letting $s(z)=r^{\prime}(z)$, we have $\omega s(\omega z)=$ $\pm s(z)$. If $\omega s(\omega z)=-s(z)$, then $-\sum_{k=0}^{m} b_{k} z^{k}=\sum_{k=0}^{m} b_{k} \omega^{k+1} z^{k}$, where $s(z)=$ $\sum_{k=0}^{m} b_{k} z^{k}$. This implies that $-b_{j}=\omega^{j+1} b_{j}$. Now $\omega^{k}=-1$ for some $k$ implies that

$$
\cos \left(\frac{2 \pi k}{N}\right)+i \sin \left(\frac{2 \pi k}{N}\right)=-1
$$

This implies $2 \pi k / N=\pi l$ for some integer $l$ and thus $\cos (2 \pi k / N)=\cos (\pi l)=1$ since $N \geq 3$ implies that $l$ is even. Hence $\omega^{k} \neq-1$ for any $k$, giving $b_{j}=0$ for all $j$. Thus $r$ is constant and so $p$ is constant and $p(z)-p(0) \in C_{0}$.

Otherwise, $\omega s(\omega z)=-s(z)$ implies that $s(\omega z)=\omega^{N-1} s(z)$, giving $s \in C_{N-1}$, and so $r \in C_{0}$, and hence $p$ and $p(z)-p(0) \in C_{0}$ as well. If $f \circ p \in C_{k}$ for some $k \geq 1$, then $f^{N} \circ p \in C_{0}$. By the case just proved, $p(z)-p(0) \in C_{k}$ for some $k$.

For the converse direction suppose that $p(z)-p(0) \in C_{k}$ for some $k$, and let $\tilde{p}(z)=p(z)-p(0)$. Then $\tilde{p}(\omega z)=\omega^{k} \tilde{p}(z)$. Let $f(z)=(z-p(0))^{N}$. Then $f(p(\omega z))=(\tilde{p}(\omega z))^{N}=\omega^{k N}(\tilde{p}(z))^{N}=(\tilde{p}(z))^{N}=(p(z)-p(0))^{N}=f(p(z))$, implying $f \circ p \in C_{0}$.

COROLlary 4.6. Let $g(z)$ be an entire function which is periodic and cyclic $(\bmod N)$ for some prime number $N \geq 3$. Then $g$ is constant. 
PROOF. Suppose that $g$ is not constant, and let $L$ be the period of $g$. Let $f(z)=$ $g(L \sqrt[N]{z})$ and $p(z)=(z+1)^{N}$. Then $f \circ p=g(L z) \in C$, but $p(z)-p(0) \notin C$, which contradicts Theorem 4.5 .

REMARK 4.5. Much of Theorem 4.3 follows from Theorem 4.5, but the latter theorem requires deeper results from [1] than used in the proof of Theorem 4.3.

\section{Rational functions}

By [3, Theorem 1] (or by Theorem 4.3 and the remark following), if $p$ and $q$ are polynomials with $q(0)=0$ and $p \circ q$ even (or odd), then $p$ or $q$ must be even (or odd). This does not hold for rational functions in general, however, as the following example shows.

EXAMPLE 5.1. Let $f(z)=g(z)=z /(z-1)$. Then $f \circ g=z$, which is odd. Also, if $f(z)=z^{2} /(z-1)^{2}$ and $g(z)=z /(z-1)$, then $f \circ g=z^{2}$, which is even. In each case, $f(0)=g(0)=0$, and neither $f$ nor $g$ are even or odd. For the cyclic case in general, just take $f(z)=z^{p} /(z-1)^{p}$ and $g(z)=z /(z-1)$.

The example above also shows that $f \circ f$ can be odd even if $f(0)=0$ and $f$ is not odd. However, $f \circ f$ cannot be even for any power series (convergent or not) if $f(0)=0$ and $f$ is not even (see [5]). The following example shows that $f \circ f$ can be even if $f(0) \neq 0$.

EXAMPLE 5.2. Let

$$
f(z)=\frac{z^{2}+z+1}{z^{2}-z+1} ; \quad \text { then } f \circ f=\frac{3 z^{4}+7 z^{2}+3}{z^{4}+5 z^{2}+1}
$$

which is even, but $f$ is not even. Note that $f$ and $f \circ f$ are both analytic at $z=0$.

\section{Polynomials in several variables}

Let $P(z, w), Q(z)$, and $R(z)$ be polynomials with $P(0,0)=Q(0)=R(0)=0$. If $P(Q(z), R(z))$ is even, must one of $P, Q$, or $R$ be even? The answer is no, as is seen by the simple example $P(z, w)=z-w^{2}, Q(z)=z^{6}+z^{4}+2 z^{3}+z^{2}, R(z)=z^{2}+z$. Then $P(Q(z), R(z))=z^{6}$, which is even, but none of $P, Q$, or $R$ are even. Note that the answer is still no if we replace even throughout by even in each variable separately. We can, however, prove the following result. We just prove the two variable case, though the extension to any number of variables follows easily. First we need the following lemma. 
LEMMA 6.1. Suppose that $P(z, w)$ is a polynomial such that $R_{a, b}(z)=P(a z, b z)$ is even for any complex constants $a$ and $b$. Then $P(z, w)$ is even.

ProOF. Suppose that $P(z, w)$ contains a homogeneous term of odd degree $k$, $\sum_{r+s=k} c_{r, s} z^{r} w^{s}$. Then the coefficient of $z^{r+s}$ in $P(a z, b z)$ is $\sum_{r+s=k} c_{r, s} a^{r} b^{s}$. Since $P(a z, b z)$ is even for any complex constants $a$ and $b$, the polynomial in $a$ and $b$, $\sum_{r+s=k} c_{r, s} a^{r} b^{s}$, is identically 0 . Then $c_{r, s}=0$ for all $r$ and $s$ such that $r+s=k$. This implies that $P(z, w)$ is even.

THEOREM 6.2. Let $R(z, w)=P(Q(z, w))$, where $P$ is a polynomial in one variable and $Q$ is a polynomial in two variables. If $Q(0,0)=0$ and $R$ is even, then $P$ or $Q$ must be even.

PROOF. Let $Q_{a, b}(z)=Q(a z, b z)$ and $R_{a, b}(z)=R(a z, b z)=P\left(Q_{a, b}(z)\right)$. Then $Q_{a, b}(0)=0$ and $R_{a, b}(z)$ is even, for any constants $a$ and $b$. By [3, Theorem 1] (or by Theorem 4.3 and the remark following), $P$ or $Q_{a, b}$ must be even. If $P$ is even, we are finished. If $P$ is not even, then $Q_{a, b}$ is even. By Lemma 6.1, $Q$ must be even.

REMARK 6.1. One can also prove an odd version of Theorem 6.2, or more generally a cyclic version, as we did for polynomials in one variable.

REMARK 6.2. Other related questions for polynomials in several variables are:

(A) If $R(z, w)=P(Q(z, w))$ is homogeneous, $Q(0,0)=0$, prove that $P$ or $Q$ must be homogeneous. This can be proved with techniques similar to those used in this paper.

(B) If $R(z, w)=P(Q(z, w))$ is symmetric, must $Q$ be symmetric?

\section{Open questions}

(1) Let $f(z)$ be an entire function with $f \circ f$ even, $f$ not even. If $f(0) \neq 0$, then $f$ need not be even (see [3]). Must $f(z)=O(z)+c$, where $O$ is odd and $c$ is a constant?

(2) Let $N \geq 3$ be a given prime number. Does there exist a function $f(z) \notin C_{0}$, analytic at 0 , such that $f \circ f \in C_{0}$ ? (If such an $f$ exists, then by [5] $f(0) \neq 0$.) If yes, does there exist a rational function $R(z) \notin C_{0}$, such that $R \circ R \in C_{0}$ ?

(3) Discuss the questions in this paper when $(f \circ g)(z)=h(k(z))$, where $h$ and $k$ are entire functions. When must $f$ or $g$ be an entire function in $k$ ?

(4) Give a complete characterization of the RPE and LPE entire functions, and in particular the LPE polynomials. 


\section{References}

[1] I. N. Baker and Fred Gross, 'On factorizing entire functions', Proc. London Math. Soc. 17 (1968), 69-76.

[2] E. Borel, 'Sur les zéros des fonctions entières', Acta Math. 20 (1897).

[3] Alan L. Horwitz and Lee A. Rubel, 'When is the composition of two power series even?', J. Austral. Math. Soc. (Series A) 56 (1994), 415-420.

[4] R. Nevanlinna, Le théorème de Picard-Borel et la théorie des fonctions méromorphes (Paris, 1929).

[5] Bruce Reznick, 'When is the iterate of a formal power series odd?', J. Austral. Math. Soc. (Series A) 28 (1979), 62-66.

Pennsylvania State University

25 Yearsley Mill Rd.

Media, PA 19063

USA

e-mail: alh4@psu.edu 